\title{
Insecticide interactions with $\gamma$-aminobutyric acid and nicotinic receptors: predictive aspects of structural models
}

\author{
John E. CASIDA* and Motohiro TOMIZAWA* \\ Environmental Chemistry and Toxicology Laboratory, Department of Environmental Science, Policy and Management, \\ University of California, Berkeley, California 94720-3112, USA
}

(Received June 21, 2007; Accepted October 9, 2007)

\begin{abstract}
An insecticide binding site may be blocked by widely diverse chemicals, as with the $\gamma$-aminobutyric acid receptor (GABAR), or accept only closely related compounds, as illustrated by the nicotinic acetylcholine (ACh) receptor (nAChR). The human GABAR $\beta 3$-homopentamer resembles the insect receptor in sensitivity and specificity for noncompetitive antagonists (NCAs), prompting exhaustive site-directed mutagenesis (cysteine scanning), which pinpointed the critical active site as pore-facing residues Ala-2', Thr-6' and Leu-9' and led to a binding site model which fits several classes of insecticidal NCAs. The nAChR agonist site was approached with an ACh binding protein (AChBP) by photoaffinity labeling and neonicotinoid docking. Chloropyridinyl chlorine contacts Ile-106/Met-116 and nitrogen is directed to Ile-118/Trp-147 via a solvent bridge(s). The guanidine/amidine plane undergoes $\pi$-stacking with Tyr-188, and the nitro/cyano pharmacophore interacts with Ser-189/Cys190, thereby defining the binding pocket that models AChBP and possibly nAChR potency and selectivity. (C) Pesticide Science Society of Japan
\end{abstract}

Keywords: agonist, $\gamma$-aminobutyric acid receptor, insecticide binding site, nicotinic acetylcholine receptor, noncompetitive antagonists, structural models.

\section{Introduction}

Neurotransmitter-gated ion channels are major targets for insecticide action, eliciting rapid intoxication for outstanding effectiveness. The $\gamma$-aminobutyric acid receptor (GABAR) and the nicotinic acetylcholine $(\mathrm{ACh})$ receptor $(\mathrm{nAChR})$ are members of this superfamily of cysteine-loop receptors which are widely distributed in the nervous systems of insects and vertebrates. Polyhalogenated insecticides, including lindane, $\alpha$-endosulfan, and fipronil, inhibit the chloride ionophore of the GABAR as noncompetitive antagonists (NCAs). Nicotine and neonicotinoid insecticides such as imidacloprid and thiacloprid are agonists of the nAChR. NCAs block the inhibitory GABAergic pathway and $\mathrm{nAChR}$ agonists directly stimulate excitatory cholinergic neurotransmission, resulting in both cases in hyperexcitation/convulsion and then paralysis. ${ }^{1,2}$ More than $20 \%$ of the current insecticides by global market

\footnotetext{
* To whom correspondence should be addressed.

E-mail: ectl@nature.berkeley.edu or tomizawa@nature. berkeley.edu

(C) Pesticide Science Society of Japan
}

value act on the GABAR and nAChR.

Knowledge of insecticide binding site interactions helps to understand the potency and selective toxicity between target insects and humans/wildlife. One target site may accept compounds of wide structural diversity and another only within a narrow range of variations. The first step involves defining structure-activity relationships (SARs) to predict the active conformation and surface charge of the insecticide and interacting fields. The second is to experimentally locate the binding site within the receptor, to incorporate it into a binding site model, and to test the model by computational docking of related or dissimilar compounds. The third goal is to apply the model to the mechanisms of target site resistance and selective toxicity. We briefly review here our progress in defining the molecular interactions of major classes of insecticides with GABAR NCA and nAChR agonist sites.

\section{GABAR NCA Site}

1.1. Human GABAR $\beta 3$ homopentamer conserves the pharmacological profile of the insect receptor

GABARs are assembled as combinations of various subunits $(\alpha, \beta, \gamma, \delta, \varepsilon, \pi, \rho$, and $\theta$ ). Recombinant receptors help define 
the relationship between subunit composition and insecticide action. ${ }^{3-5)}$ The potency of insecticides acting at the GABAR NCA site in the chloride ion channel can be predicted by binding assays with $4^{\prime}$-ethynyl-4- $n-\left[1,2-{ }^{3} \mathrm{H}\right]$ propylbicycloorthobenzoate $\left(\left[{ }^{3} \mathrm{H}\right] \mathrm{EBOB}\right)$, which also shows high insecticidal activity. A $\beta$ subunit is required alone or with another subunit for assembly of the radioligand binding site; all recombinant receptors without a $\beta$ subunit (except $\rho 1$ homopentamer) give no specific $\left[{ }^{3} \mathrm{H}\right] \mathrm{EBOB}$ binding. $\beta 1$-containing receptors and the $\rho 1$ homopentamer are much less sensitive than $\beta 3$-containing receptors to NCAs (Table 1). The $\beta 3$ homopentameric GABAR is readily expressed and functional. Co-assembly of $\beta 3$ with $\alpha 1, \alpha 6$, or $\gamma 2$ subunits clearly reduces the affinity of NCAs, as observed also for the human native receptor. We discovered that the human $\beta 3$ homopentameric receptor is similar to the insect GABAR in insecticide sensitivity and specificity (Table 1).

\subsection{Three amino acids important to NCA insecticide binding}

The first step in pinpointing the NCA insecticide binding site was identification of the molecular determinant for target site resistance. The Ala-2' to Ser-2' mutation (Fig. 1) confers cy-

Table 1. Effect of human recombinant GABAR subunit composition versus native human and insect receptors on binding potencies of $\alpha$-endosulfan, lindane, and fipronil ${ }^{a)}$

\begin{tabular}{lccc}
\hline \multirow{2}{*}{ Receptor } & \multicolumn{3}{c}{$\mathrm{IC}_{50}(\mathrm{nM})$} \\
\cline { 2 - 4 }$\alpha$-Endosulfan $^{b)}$ & Lindane $^{b)}$ & Fipronil $^{b)}$ \\
\hline Recombinant with $\beta 3$ subunit & & \\
$\beta 3$ & 0.47 & 0.90 & 2.4 \\
$\alpha 6 \beta 3$ & 4.0 & 1.3 & 3.1 \\
$\alpha 6 \beta 3 \gamma 2$ & 4.6 & 4.9 & 17 \\
$\alpha 1 \beta 3$ & 8.3 & 12 & 28 \\
$\alpha 1 \beta 3 \gamma 2$ & 16 & 21 & 33 \\
Recombinant with $\beta 1$ subunit & 117 & 112 & 7700 \\
$\alpha 1 \beta 1$ & 40 & 67 & 2800 \\
$\alpha 1 \beta 1 \gamma 2$ & 800 & 91 & $>10000$ \\
Recombinant with $\rho 1$ subunit & & \\
$\rho 1$ & & & 2.3 \\
Native & & & \\
Human & & & \\
Insect & 7.3 & 11 & 2506 \\
\hline
\end{tabular}

a) Illustrative data from Refs. 3-5. ${ }^{b)} \mathrm{LD}_{50}(\mathrm{mg} / \mathrm{kg})$ values of $\alpha$-endosulfan, lindane, and fipronil are 10, 40, and 32, respectively, for mouse (intraperitoneal route) and 5.5, 5.5, and 0.25 for housefly (topical application). ${ }^{3)}{ }^{c)} \mathrm{IC}_{50}$ human $\mathrm{GABAR} / \mathrm{IC}_{50}$ insect receptor: $\alpha$-endosulfan 0.73 ; lindane 28 ; fipronil 1090 . In comparison, the affinity ratio for EBOB is 5.0.,

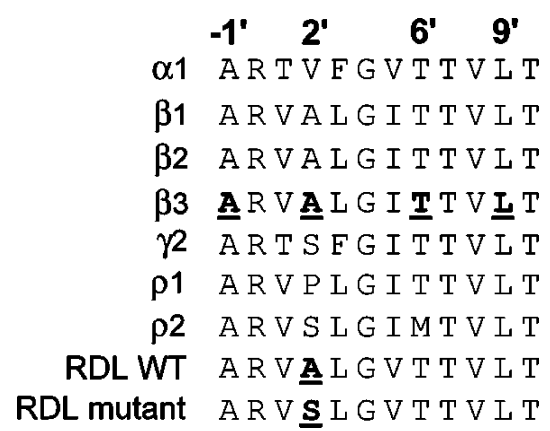

Fig. 1. GABAR subunit sequence homologies. Alignment of the cytoplasmic half of the transmembrane 2 (TM2) region. The species are human or rat for $\alpha, \beta, \gamma$, or $\rho$, Drosophila for the wild type (WT), and Drosophila for a resistant-to-dieldrin (RDL) mutant. Index numbers for positioning in TM2 are shown at the top. Porefacing residues $\left(-1^{\prime}, 2^{\prime}, 6^{\prime}\right.$, and $\left.9^{\prime}\right)$ in the $\beta 3$ homopentamer are shown underlined in bold. The resistance-associated RDL mutant (Ala to Ser at $\left.2^{\prime}\right)^{6)}$ is also shown underlined in bold.

clodiene resistance in a broad range of insects. ${ }^{6}$ In the $\beta 3$ homopentameric channel, the 12 amino acids shown in Fig. 1 were mutated to Cys, Ser, or other residues. ${ }^{7)}$ Positions $2^{\prime}, 6^{\prime}$, and $9^{\prime}$ were localized in the channel lumen (Fig. 2) by disulfide cross-linking. Binding of two NCA radioligands, EBOB and 3,3-bis-trifluoromethylbicyclo[2.2.1] heptane-2,2-dicarbonitrile (BIDN), was greatly reduced with mutations at these three positions, focusing attention on Ala-2', Thr- $6^{\prime}$, and Leu$9^{\prime}$ as the proposed binding sites, consistent with mutagenesis studies in other systems. ${ }^{8)}$ Five other mutations $\left(-1^{\prime}, 0^{\prime}, 4^{\prime}\right.$, $5^{\prime}$, and $8^{\prime}$ except $3^{\prime}$ not expressed) in the $-1^{\prime}$ to $10^{\prime}$ region also impaired radioligand binding, suggesting conformational changes in the channel wall.

\subsection{Structural model for GABAR-NCA interactions with structurally diverse insecticides}

The first substantial GABAR model was established with the heteropentameric $\alpha 1 \beta 2 \gamma 2$ subtype. ${ }^{9)}$ Homopentameric human $\beta 3$ GABAR is very similar to that of insect GABAR in sensitivity to insecticides and general amino acid sequence of the TM2 region (Fig. 1). With the same sequence for each subunit, the $\beta 3$ homopentamer is assembled in completely symmetrical form, greatly simplifying the modeling of ligand-receptor interactions. The pore size of $8.5 \AA$ is appropriate for binding only a single NCA molecule. This model leads to the novel conclusion that NCAs with widely diverse chemical structures fit the same binding site and block the channel illustrated with EBOB, lindane, $\alpha$-endosulfan, fipronil, and picrotoxinin in Fig. 2, with the addition of BIDN and the EBOB analog TBPS in the original report. ${ }^{7)}$ The interacting residues are Ala- $2^{\prime}$ (or more generally the Ala- $2^{\prime}$ to Ile- $5^{\prime}$ hydrophobic pocket) and Thr- $6^{\prime}$ (the most important molecular determinant) with a supplemental role for Leu- $9^{\prime}$. The more compact NCAs require only Ala- $2^{\prime}$ and Thr-6', whereas the potency of long or extended NCA molecules is enhanced by the Leu-9' 


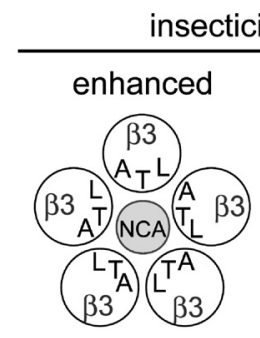

$(\beta 3)_{5}$ homopentamer

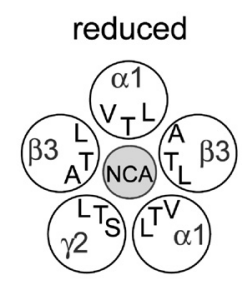

$(\alpha 1)_{2}(\beta 3)_{2} \gamma 2$

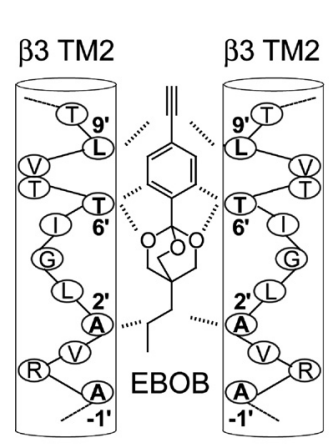

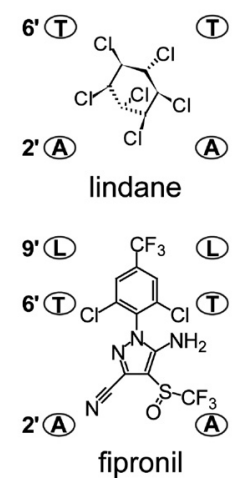
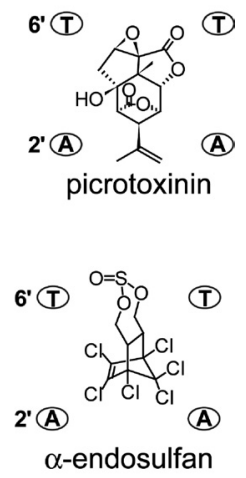

Fig. 2. GABAR NCA site. Multiple subunit combinations forming the GABAR chloride ionophore confer unique sensitivity to NCA insecticides attributable in part to three pore-facing residues at $2^{\prime}$ (A, $\mathrm{S}$, or $\mathrm{V}), 6^{\prime}(\mathrm{T})$, and $9^{\prime}$ (L) (Upper). Molecular interactions are shown for five NCAs at the cytoplasmic end of the TM2/ $\alpha$-helix of the human $\beta 3$ homopentameric channel (Lower). ${ }^{7)}$ Two of the five $\beta 3$ subunits are illustrated. These schemes do not allow visualization of three-dimensional positions and geometries for NCAs and their interacting residues.

side chain. Favorable GABAR-NCA interactions (hydrophobic and hydrogen bonding) are illustrated in Fig. 2 and detailed by Chen et al. ${ }^{7)}$ The model provides a template for ligand design, structure optimization and improved potency. Consideration of known SARs shows that high affinity corresponds to binding in a more stable configuration with
$>30 \mathrm{~kJ} / \mathrm{mol}$ differences for the more potent $\gamma$ (lindane) than the less active $\alpha, \beta$ and $\gamma$ isomers of hexachlorocyclohexane, and the more potent $\alpha$ than the less active $\beta$-endosulfan. A correlation between affinity and total binding energy in a docking model was also observed for fipronil analogs with the $\beta 3$ homopentamer. ${ }^{10)}$

\section{Nicotinic Agonist Site}

\subsection{ACh binding protein $(A C h B P)$ as a structural surro- gate for $n A C h R s$}

The structural and functional architecture of Torpedo nAChR was established by electron microscopy ${ }^{11)}$ but not with adequate resolution to understand the recognition properties of the insecticide binding sites. These sites are localized in interface regions between subunits, and specific subunit combinations confer differences in sensitivity to ACh and in pharmacological profiles. Knowledge of drug-nAChR interactions was greatly advanced by studies on soluble AChBPs from Aplysia sp. and other molluscs as structural surrogates for the extracellular ligand-binding domain of nAChR (Fig. 3). ${ }^{12-14)}$ The nAChR is hetero- or homopentameric with two or five ACh binding sites, respectively, while AChBP with a defined high resolution crystal structure is a homopentamer with five agonist binding sites.

\subsection{Photoaffinity labeling of $A C h B P$}

Neonicotinoids have a chloropyridinyl or equivalent moiety and are nonprotonated with a nitro- or cyano-substituted guanidine/amidine pharmacophore. ${ }^{15)}$ Site-directed mutagenesis has recognized several amino acids which potentially modulate neonicotinoid agonist action. ${ }^{16,17)}$ Photoaffinity labeling is an alternative and direct approach for structural investigation of the target. Azidochloropyridinyl neonicotinoids are candidate photoaffinity probes. ${ }^{18,19)}$ If they bind to the specific site, the reactive nitrene generated by photoirradiation should derivatize AChBP, allowing identification of modified amino acid residue(s) by mass spectrometry. The neonicotinoid probes specifically photoreacted with Aplysia AChBP, which is sensitive to neonicotinoids, in an incorporation ratio of one acti-
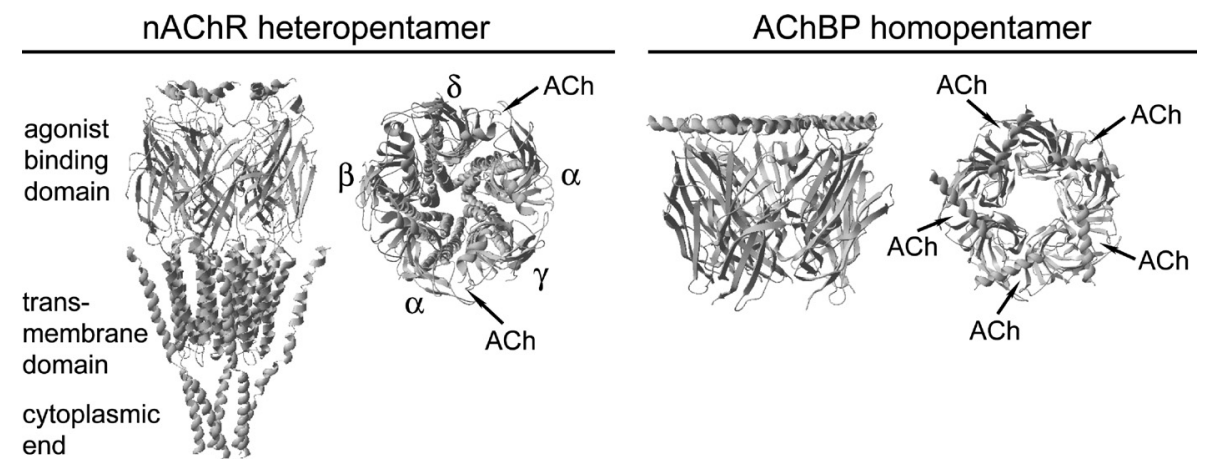

Fig. 3. nAChR and AChBP comparison. Overall structures of Torpedo heteropentameric nAChR and Aplysia homopentameric AChBP [views from the side (left) or top (right) of each protein] based on electron microscopy and X-ray crystal data, respectively. ${ }^{11,14)}$ AChBP does not have a transmembrane domain or cytoplasmic end. Agonist binding pockets are localized at subunit interfaces. 

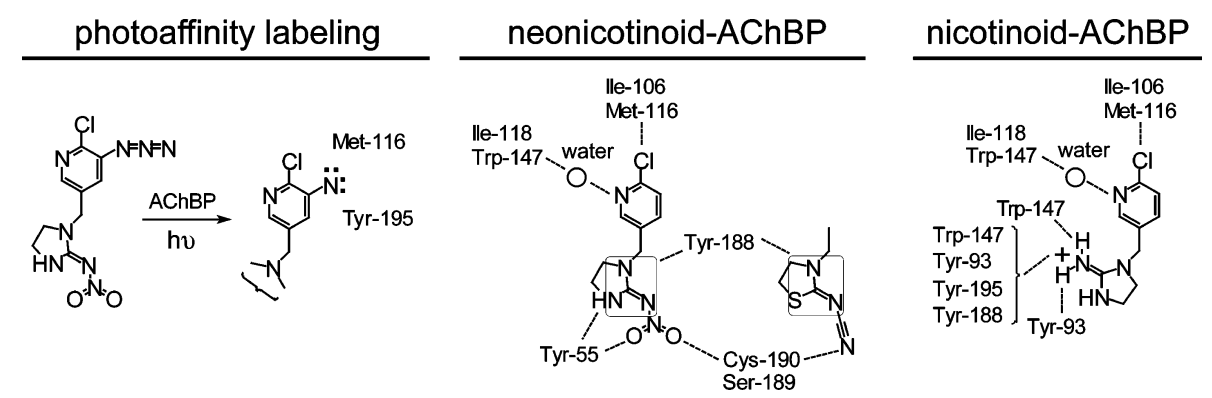

Fig. 4. AChBP binding site. ${ }^{20)}$ Photoaffinity labeling with azidoimidacloprid as a representative probe and the photoactivated nitrene that modifies Met-116 and Tyr-195 at the subunit interface (Left). Schematic representations of AChBP-neonicotinoid (Middle) and AChBP-nicotinoid (Right) interactions. Chloropyridinyl neonicotinoids imidacloprid and thiacloprid with nitro- and cyano-substituted guanidine and amidine moieties, respectively (as indicated by boxes), are compared with the nicotinoid desnitro analog of imidacloprid with the inverted guanidinium pharmacophore. Tyr-195 is not shown in the middle scheme because it does not interact with the nitro/cyano neonicotinoid pharmacophore. These schemes do not reflect three-dimensional positions and geometries in the ligand binding pocket.

vated nitrene molecule in each subunit interfacial binding domain (Fig. 4). The sites for specific derivatization were Tyr195 and Met-116 in similar frequency. ${ }^{20)}$ Tyr-195 is located on loop $\mathrm{C}$ of the principal or (+)-face subunit of AChBP, whereas Met-116 is on loop E of the complementary or (-)-face subunit and these residues are three-dimensional neighbors.

\subsection{AChBP as a structural model for neonicotinoid and nicotinoid interactions}

A structural model was established by combining $(i)$ the crystal structure of Aplysia AChBP alone and complexed with the chloropyridinyl nicotinoid analog epibatidine, (ii) photoaffinity labeling for precisely locating the chloropyridinyl moiety, and (iii) predicted positioning of the rest of the molecule (Fig. 4). ${ }^{20)}$ The chlorine of imidacloprid and thiacloprid makes van der Waals contacts with loop E, particularly the backbone carbonyl oxygens of Ile-106 and Met-116. The pyridinyl nitrogen hydrogen bonds with the carbonyl oxygens of Ile-118 and Trp-147 via solvent bridge(s). The $\mathrm{OH}$ oxygen of Tyr-55 contacts the imidazolidine $\mathrm{NH}$ hydrogen of imidacloprid. The guanidine/amidine plane of the two neonicotinoids binds with Tyr-188 in a slipped-stack geometry for $\pi$-interaction. The important electronegative oxygen or cyano nitrogen tip hydrogen bonds with the backbone $\mathrm{NH}$ of Cys-190. The other nitro oxygen of imidacloprid also interacts with the $\mathrm{OH}$ hydrogen of Tyr-55. Further hydrogen bonding and/or hydrophobic contacts are predicted between the neonicotinoid tip atom (nitro O or cyano N) and Cys-190 and Ser-189. The conformational flexibility of loop $\mathrm{C}$ induced by agonist binding ${ }^{14)}$ plays an important role in neonicotinoid interactions. ${ }^{20)}$ The desnitro and descyano derivatives of neonicotinoids, as with epibatidine, are positively charged (iminium or ammonium cation) and prefer the mammalian receptor. Their azido derivatives as photoaffinity probes showed the same positioning of the chloropyridinyl moiety for neonicotinoids and nicotinoids. ${ }^{20,21)}$ Molecular modeling revealed an inverted pharmacophore relationship of neonicotinoids and nicotinoids, as shown in Fig. 4.

\section{Toxicological Implications of Structural Models}

\subsection{Experimental basis for structural models}

Two very different approaches were taken in preparing structural models for GABAR-NCA and nAChR-neonicotinoid interactions. The structure of GABAR $\alpha$-helical TM2 is suitable for the site-directed mutagenesis (scanning) approach, serving to recognize crucial contributors to NCA binding. The $\beta 3$ homopentamer provides identical amino acid linings in the ion channel lumen from each of five subunits and conserves the NCA site in its most sensitive form. On the other hand, the extracellular agonist binding domain of nAChR consists of several discontinuous loops and $\beta$-sheets. The high resolution crystal structure of soluble AChBP serves as an excellent model for $\mathrm{nAChR}$ in characterizing the localization and geometry of amino acid side chains in the agonist-binding site. ${ }^{12-14)}$ In addition, photoaffinity labeling defines the precise positioning of the agonist in physiological conditions where the protein is in solution or at the membrane. Therefore, we developed and used these two structural models for predicting receptor-insecticide interactions relevant to resistance mechanisms and selective toxicity.

\subsection{Resistance mechanisms}

The GABAR Ala-2' to Ser-2' mutation (Fig. 1) confers high insecticide resistance and low GABAR sensitivity ${ }^{6}$ with varying degrees of cross resistance to diverse types of NCAs. The relative importance of the $2^{\prime}, 6^{\prime}$ and $9^{\prime}$ sites in insecticide binding may contribute to differences in resistance levels, e.g. the importance of $9^{\prime}$ for fipronil and EBOB. Several other mutated $\beta 3$ homopentamer TM2 sites also reduce NCA binding $^{7)}$ and a TM3 mutation in Drosophila confers resistance to fipronil. $^{22)}$ A nAChR leafhopper mutation associated with lower imidacloprid mortality and receptor sensitivity than the wild type involves Tyr-151 to Ser-151 (adjacent to loop B Trp-149) of $\alpha$ subunits. This mutation influences the agonist 
potency of several chloropyridinyl or chlorothiazolyl neonicotinoids and epibatidine but not of two non-chlorinated analogs (nicotine and the tetrahydrofuryl dinotefuran) or $\mathrm{ACh}^{23,24)}$ Based on analogy to the AChBP model, this single mutation may induce conformational change in the area between loops $\mathrm{B}$ and $\mathrm{E}$ where it is in close proximity to the binding position for the neonicotinoid chloropyridinyl/ chlorothiazolyl ring. Interestingly, a similar pattern was noted for another leafhopper with much higher sensitivity to tetrahydrofuryl compounds and $\mathrm{ACh}$ when assayed with $\left[{ }^{3} \mathrm{H}\right]$ dinotefuran than with $\left[{ }^{3} \mathrm{H}\right]$ chloropyridinyl radioligands (imidacloprid and acetamiprid). ${ }^{25)}$

\subsection{Selectivity toxicity}

Insecticides acting at the GABAR NCA site range from essentially nonselective to highly insecticidal with moderate toxicity to mammals. The native receptor insect/human target site selectivity ratio decreases in the order fipronil $>$ lindane $>$ endosulfan or EBOB (shown partially in Table 1). The affinity difference among these compounds is very large with the human native receptor compared with the recombinant $\beta 3$ homopentamer. Thus, subunit composition may be a central feature in conferring NCA selectivity. Insecticide sensitivity is greatly enhanced for the $\beta 3$ homopentamer model compared with the $\alpha 1 \beta 3 \gamma 2$ heteropentamer, possibly due in part to varied pore-facing residues at the $2^{\prime}$ position (Figs. 1 and 2) More generally, the $\beta$ subunit, particularly $\beta 3$, directly contributes to insecticide interaction, and other subunits differentially modulate binding affinity to confer compound-dependent specificity and selectivity, i.e., unique pharmacologies. Insect nAChR is highly sensitive to neonicotinoids and mammalian nAChR to nicotinoids. The AChBP model suggests that neonicotinoid affinity is due to the partially electronegative nitro or cyano substituent important in conferring unique fit at the loop $\mathrm{C}$ region. The neonicotinoid pharmacophore involves the nitro- or cyano-substituted guanidine/amidine coplanar system which binds in an inverted position relative to the nicotinoid cationic functionality. These types of neonicotinoid and nicotinoid interactions with AChBP subsites may serve as models for their unique positioning with nAChRs of insects and mammals, contributing to an understanding of selective agonist action and toxicity. In conclusion, these insecticide binding site structural and functional models provide precedents for the discovery and understanding of new compounds with outstanding effectiveness and maximal safety.

\section{Acknowledgments}

We thank our Berkeley laboratory alumni Ligong Chen, Gurpreet Ratra, Nanjing Zhang, and Shinzo Kagabu and collaborators from other institutions who contributed to the concepts and findings. J. E. C. acknowledges support from the William Muriece Hoskins Chair in Chemical and Molecular Entomology. J. E. C. and M. T. are supported by NIH NIEHS grant R01 ES008424.

\section{References}

1) J. E. Casida and G. B. Quistad: Annu. Rev. Entomol. 43, 1-16 (1998).

2) J. E. Casida and G. B. Quistad: J. Pestic. Sci. 29, 81-86 (2004).

3) G. S. Ratra, S. G. Kamita and J. E. Casida: Toxicol. Appl. Pharmacol. 172, 233-240 (2001).

4) G. S. Ratra and J. E. Casida: Toxicol. Lett. 122, 215-222 (2001).

5) G. S. Ratra, B. E. Erkkila, D. S. Weiss and J. E. Casida: Toxicol. Lett. 129, 47-53 (2002).

6) R. H. ffrench-Constant, N. Anthony, K. Aronstein, T. Rocheleau and G. Stilwell: Annu. Rev. Entomol. 45, 449-466 (2000).

7) L. Chen, K. A. Durkin and J. E. Casida: Proc. Natl. Acad. Sci. U.S.A 103, 5185-5190 (2006).

8) R. W. Olsen: Proc. Natl. Acad. Sci. U.S.A. 103, 6081-6082 (2006).

9) M. O'Mara, B. Cromer, M. Parker and S.-H. Chung: Biophys. J. 88, 3286-3299 (2005).

10) M. S. Alam, J. Huang, F. Ozoe, F. Matsumura and Y. Ozoe: Bioorg. Med. Chem. 15, 5090-5104 (2007).

11) N. Unwin: J. Mol. Biol. 346, 967-989 (2005).

12) K. Brejc, W. J. van Dijk, R. V. Klaassen, M. Schuurmans, J. van der Oost, A. B. Smit and T. K. Sixma: Nature 411, 269-276 (2001).

13) P. H. N. Celie, S. E. van Rossum-Fikkert, W. J. van Dijk, K. Brejc, A. B. Smit and T. K. Sixma: Neuron 41, 907-914 (2004).

14) S. B. Hansen, G. Sulzenbacher, T. Huxford, P. Marchot, P. Taylor and Y. Bourne: EMBO J. 24, 3635-3646 (2005).

15) M. Tomizawa and J. E. Casida: Annu. Rev. Pharmacol. Toxicol. 45, 247-268 (2005).

16) M. Shimomura, M. Yokota, K. Matsuda, D. B. Sattelle and K Komai: Neurosci. Lett. 363, 195-198 (2004).

17) M. Shimomura, M. Yokota, M. Ihara, M. Akamatsu, D. B. Sattelle and K. Matsuda: Mol. Pharmacol. 70, 1255-1263 (2006).

18) S. Kagabu, P. Maienfisch, A. Zhang, J. Granda-Minones, J. Haettenschwiler, H. Kayser, T. Maetzke and J. E. Casida: J. Med. Chem. 43, 5003-5009 (2000).

19) N. Zhang, M. Tomizawa and J. E. Casida: J. Med. Chem. 45, 2832-2840 (2002).

20) M. Tomizawa, T. T. Talley, D. Maltby, K. A. Durkin, K. F. Medzihradszky, A. L. Burlingame, P. Taylor and J. E. Casida: Proc. Natl. Acad. Sci. U.S.A. 104, 9075-9080 (2007).

21) M. Tomizawa, D. Maltby, K. F. Medzihradszky, N. Zhang, K. A. Durkin, J. Presley, T. T. Talley, P. Taylor, A. L. Burlingame and J. E. Casida: Biochemistry 46, 8798-8806 (2007).

22) G. Le Goff, A. Hamon, J.-B. Berge and M. Amichot: J. Neurochem. 92, 1295-1305 (2005).

23) Z. Liu, M. S. Williamson, S. J. Lansdell, I. Denholm, Z. Han and N. S. Millar: Proc. Natl. Acad. Sci. U.S.A. 102, 8420-8425 (2005).

24) Z. Liu, M. S. Williamson, S. J. Lansdell, Z. Han, I. Denholm and N. S. Millar: J. Neurochem. 99, 1273-1281 (2006).

25) H. Honda, M. Tomizawa and J. E. Casida: J. Agric. Food Chem. 54, 3365-3371 (2006). 